Streptococcus pneumoniae antimicrobial resistance decreased in the Helsinki Metropolitan Area after routine 10-valent pneumococcal conjugate vaccination of infants in Finland

Sihvonen, R.

2017-11

Sihvonen , R , Siira , L , Toropainen , M , Kuusela , P \& Patari-Sampo , A 2017 , '

Streptococcus pneumoniae antimicrobial resistance decreased in the Helsinki Metropolitan Area after routine 10-valent pneumococcal conjugate vaccination of infants in Finland ', European Journal of Clinical Microbiology \& Infectious Diseases, vol. 36 , no. 11, pp. 2109-2116 . https://doi.org/10.1007/s10096-017-3033-5

http://hdl.handle.net/10138/260145

https://doi.org/10.1007/s10096-017-3033-5

publishedVersion

Downloaded from Helda, University of Helsinki institutional repository.

This is an electronic reprint of the original article.

This reprint may differ from the original in pagination and typographic detail.

Please cite the original version. 


\title{
Streptococcus pneumoniae antimicrobial resistance decreased in the Helsinki Metropolitan Area after routine 10-valent pneumococcal conjugate vaccination of infants in Finland
}

\author{
R. Sihvonen ${ }^{1}$ (D) $\cdot$ L. Siira ${ }^{2} \cdot$ M. Toropainen ${ }^{2} \cdot{\text { P. } \text { Kuusela }^{1} \cdot \text { A. Pätäri-Sampo }}^{1}$
}

Received: 5 February 2017 / Accepted: 29 May 2017 /Published online: 13 June 2017

(C) Springer-Verlag Berlin Heidelberg 2017

\begin{abstract}
Since the introduction of 10-valent pneumococcal conjugate vaccine (PCV10) into the Finnish national vaccination program in September 2010, the incidence of invasive pneumococcal disease in children has decreased steeply in Finland. We studied the antimicrobial susceptibility of invasive and non-invasive Streptococcus pneumoniae (pneumococcus) isolated in the Helsinki Metropolitan Area during 2009-2014. We divided the data into two age groups: isolates from patients $<5$ years old and $\geq 5$ years old. We also studied the serotype distribution of invasive isolates and of a subset of non-invasive multidrug-resistant isolates. The invasive isolate numbers recovered from patients aged $<5$ years old declined from 33/228 (15\%) in 2009 to $8 / 208$ (4\%) in $2014(p<0.001)$ and non-invasive isolate numbers declined during the same time period from $221 / 595$ (37\%) to $119 / 432$ $(28 \%)(p<0.001)$. At the same time, the proportion of penicillin non-susceptible non-invasive isolates in this age group decreased from $25 \%(56 / 220)$ to $13 \%(15 / 119)(p=0.001)$ and multidrug-resistant isolates from $22 \%(49 / 220)$ to $6 \%(7 / 119)$ $(p<0.001)$, respectively. The number of PCV10 serotype isolates also decreased among the serotyped multidrugresistant non-invasive isolates. Among patients aged $\geq 5$ years old, the isolate numbers did not show a similar decreasing trend compared to the younger group and, further, the number of non-PCV10 serotype isolates increased in invasive cases.
\end{abstract}

R. Sihvonen

reetta.sihvonen@hus.fi

1 Department of Bacteriology, University of Helsinki and Helsinki University Hospital, HUSLAB, Haartmaninkatu 3, 00290 Helsinki, Finland

2 Department of Infectious Diseases, National Institute for Health and Welfare, Mannerheimintie 166, 00300 Helsinki, Finland
To conclude, the antimicrobial non-susceptibility of pneumococcus has decreased markedly, especially among young patients $(<5$ years old), following PCV10 implementation in Finland.

\section{Introduction}

Streptococcus pneumoniae (pneumococcus) causes noninvasive respiratory tract infections and invasive infections, such as bacteremic pneumonia, septicemia, and meningitis [1]. Antimicrobial agents are essential in treating pneumococcal infections and, before penicillin treatment became available, the mortality of pneumococcal bacteremia was over $80 \%$ [2]. Until the late 1970s, pneumococcus was susceptible to penicillin and other beta-lactam antimicrobials, but during the last several decades, the rise in antimicrobial resistance has become a global concern [3]. During the last decade, the proportion of non-susceptible invasive isolates has also risen in Finland. In 2010, 23\% of the isolates were penicillin nonsusceptible [minimum inhibitory concentration $(\mathrm{MIC}) \geq 0.12 \mathrm{mg} / \mathrm{L})[4,5]$.

The polysaccharide capsule is the major pneumococcal virulence factor and over 90 pneumococcal capsular serotypes are currently recognized [6]. A pneumococcal conjugate vaccine (PCV) against seven common serotypes (PCV7) causing invasive disease in children has been available since 2000 in the USA and since 2001 in Europe, and its effectiveness against invasive pneumococcal disease (IPD), especially in young children, has been well documented [7-10]. Later, PCVs covering ten (PCV10) or thirteen (PCV13) serotypes have been developed [11] and introduced into national vaccination programs, replacing PCV7.

During 2009-2010, a large cluster-randomized PCV10 trial was conducted in Finland showing the effectiveness of 
PCV10 against both culture-confirmed and clinically suspected IPD [12, 13]. In September 2010, after a public tender, PCV10 was introduced into the national childhood vaccination program in Finland using a $2+1$ schedule. Since then, the overall incidence of IPD in vaccine-eligible children has decreased by $80 \%$; the incidence of IPD among unvaccinated children has also decreased, suggesting indirect protection [14]. The vaccination coverage has been high; approximately $95 \%$ of young children have received the full three-dose series of vaccine, based on the statistics from the years 2010 and 2011 [15].

The aim of the study was to investigate changes in the antimicrobial resistance patterns of invasive and noninvasive clinical pneumococcal isolates collected from the Helsinki Metropolitan Area of approximately 1.8-2 million inhabitants during 2009-2014. We also studied the serotype distribution of the invasive isolates and of a subset of noninvasive multidrug-resistant (MDR) isolates to assess possible serotype replacement.

\section{Materials and methods}

\section{Isolates and species identification}

Clinical invasive (isolated from the blood or the cerebrospinal fluid) and non-invasive (all others, e.g., from ear, eye, nose, throat, maxillary sinus, trachea, bronchus, sputum, or abscess) S. pneumoniae isolates from the years 2009-2014 were routinely identified at HUSLAB (Hospital District of Helsinki and Uusimaa Laboratory Services) using optochin sensitivity testing or matrix-assisted laser desorption/ionization time-offlight mass spectrometry (VITEK MS MALDI-TOF, bioMérieux SA, Marcy l'Etoile, France). Only the first isolate of the specific year per patient was included in the study. Both invasive and non-invasive isolates were divided into two age groups: isolates from patients $<5$ years old and $\geq 5$ years old. The study design is shown in Fig. 1. Of the invasive samples positive for $S$. pneumoniae, 92\% (1158/1254) were positive by culture and the remaining $8 \%(96 / 1254)$ by the detection of nucleic acid (AccuProbe or lytA-specific PCR [16]).

\section{Antimicrobial susceptibility testing}

Susceptibility testing was performed routinely by the European Committee on Antimicrobial Susceptibility Testing (EUCAST) methodology using 2014 breakpoints to interpret the zone inhibitions or MIC breakpoints as susceptible (S), intermediate (I), or resistant (R). For invasive isolates, susceptibilities to penicillin, erythromycin, clindamycin, ceftriaxone, telithromycin, levofloxacin, and moxifloxacin were mainly determined. For non-invasive isolates, susceptibilities to penicillin, erythromycin, clindamycin, tetracycline, and

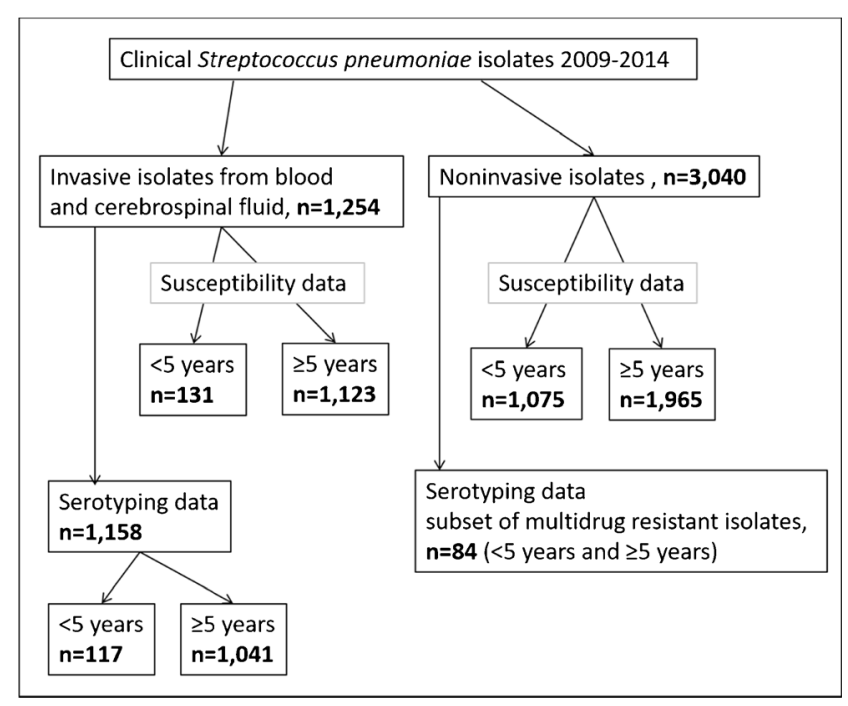

Fig. 1 Study design

trimethoprim-sulfamethoxazole were determined. For all isolates, breakpoints for infections other than meningitis were used for benzylpenicillin (MIC S $\leq 0.06 \mathrm{mg} / \mathrm{L}, \mathrm{R}>2 \mathrm{mg} / \mathrm{L}$ ), so for this study, invasive and non-invasive isolates were categorized equally concerning SIR. Figures 2 and 4 show the actual numbers of tested isolates for each antibiotic. Testing was done primarily by disk diffusion (Oxoid, Cambridge, UK) and gradient tests (Etest, bioMérieux SA, Marcy l'Etoile, France), when appropriate. An oxacillin disk was used to screen penicillin susceptibility and the penicillin susceptibility was verified with the gradient test when the zone diameter was smaller than $20 \mathrm{~mm}$. Multidrug resistance was defined as non-susceptibility (I or R) to penicillin coupled with resistance to a minimum of two other antimicrobial classes aside from beta-lactams.

\section{Serotyping}

All available invasive isolates $(n=1158)$ were serotyped at the reference laboratory at the National Institute for Health and Welfare (THL) by multiplex polymerase chain reaction (PCR), latex agglutination, counterimmunoelectrophoresis, or Quellung reaction [17]. Serotype data of invasive isolates have been used partly in previous studies [4, 14]. For this study, all available non-invasive MDR isolates $(n=84)$ from the years 2009-2014 were serotyped by multiplex PCR supplemented with Quellung reaction, when needed. The MDR isolates represented all $S$. pneumoniae MDR isolates from the study years that were stored at HUSLAB and included isolates from both age groups ( $<5$ and $\geq 5$ years). The serotype data were divided into PCV10 serotypes (serotypes 1, 4, 5, 6B, 7F, $9 \mathrm{~V}, 14,18 \mathrm{C}, 19 \mathrm{~F}$, and 23F) and non-PCV10 serotypes (all other serotypes). 

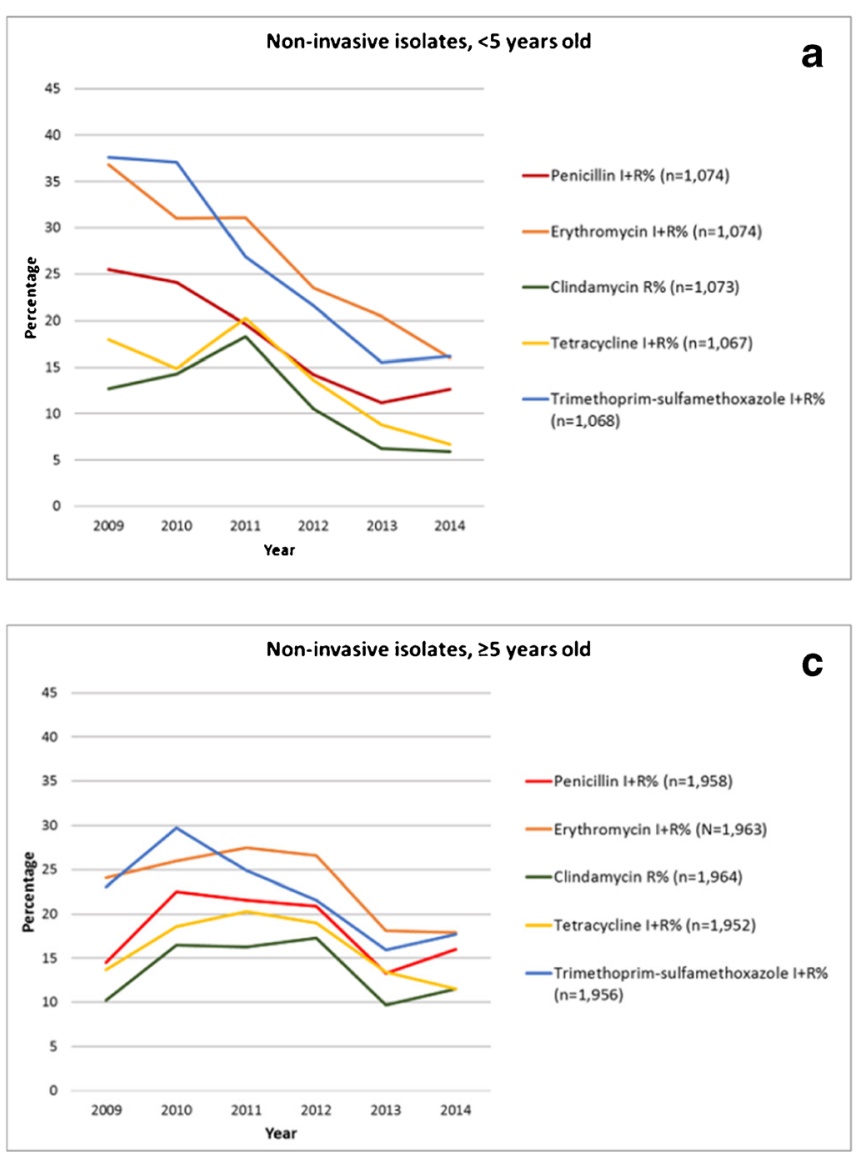

Fig. 2 a The proportion of non-susceptible non-invasive Streptococcus pneumoniae isolates in the $<5$ years age group by year. $\mathbf{b}$ The proportion of multidrug-resistant $(M D R)$ and non-multidrug-resistant (non-MDR) non-invasive $S$. pneumoniae isolates in the $<5$ years age group. c The

\section{Statistical analysis}

The differences in the antimicrobial non-susceptibility or serotype distribution between the study years were calculated using the Chi-square test by SPSS Statistics ver. 22.0 (IBM Co., Armonk, NY, USA). All six years (2009-2014) were included for analysis and a $p$-value $<0.05$ was considered statistically significant.

\section{Results}

During 2009-2014, a total of 3040 non-invasive and 1254 invasive $S$. pneumoniae isolates were characterized at HUSLAB. The median age of the non-invasive and invasive patients was 23 years (range $0-94$ ) and 59 years (range 0 100), respectively. During the study years from 2009 to 2014 , the number of non-invasive isolates declined by $46 \%$ and $16 \%$ in the $<5$ years and $\geq 5$ years age groups, respectively $(p<0.001$ for both). The invasive isolate number declined by
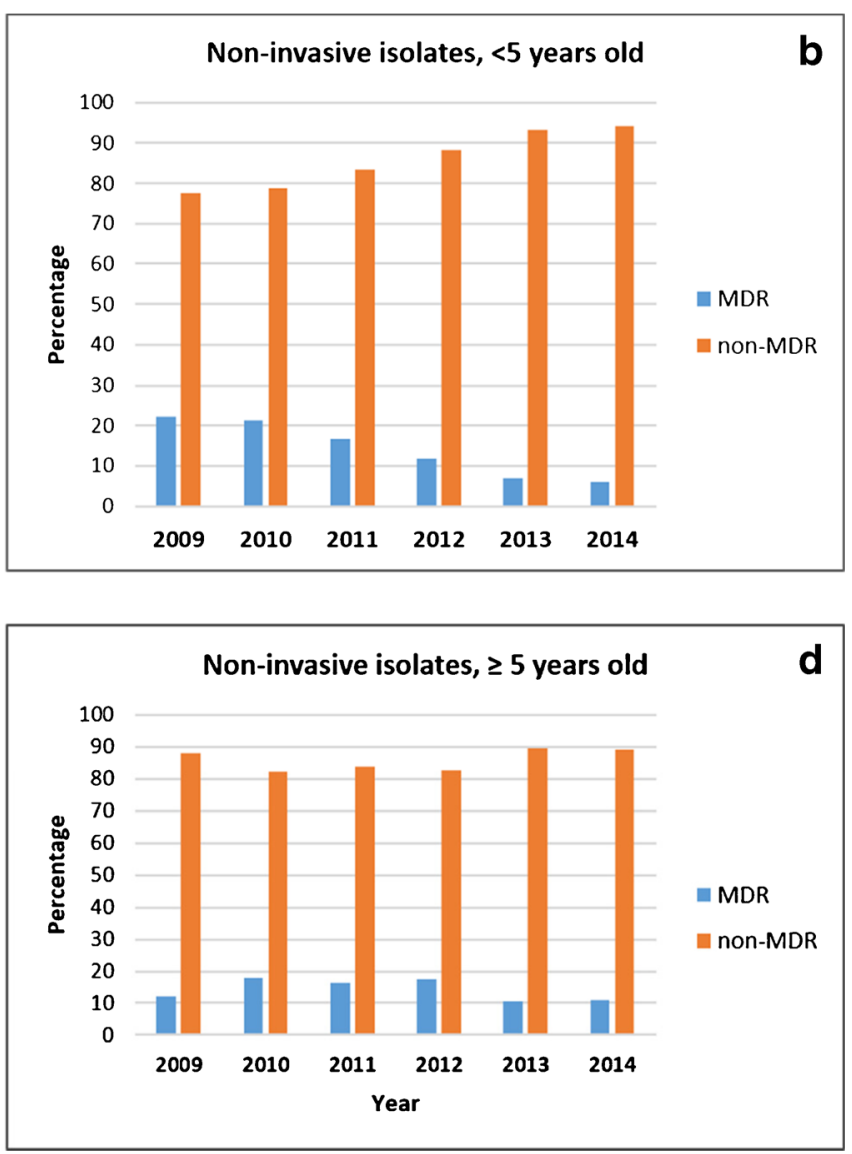

proportion of non-susceptible non-invasive S. pneumoniae isolates in the $\geq 5$ years age group by year. $\mathbf{d}$ The proportion of multidrug-resistant $(M D R)$ and non-multidrug-resistant (non-MDR) non-invasive $S$. pneumoniae isolates in the $\geq 5$ years age group

$76 \%$ in the $<5$ years age group but increased by $2.5 \%$ in the $\geq 5$ years age group $(p<0.001$ for both). Non-invasive isolate numbers are presented in Table 1 and invasive isolate numbers in Table 2.

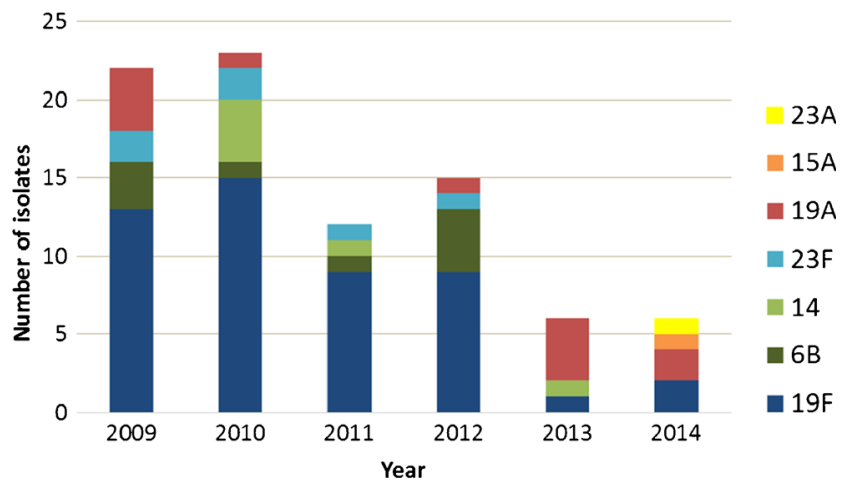

Fig. 3 Serotypes of a subset of non-invasive multidrug-resistant isolates $(n=84)$. Both age groups were included. PCV10 serotypes: 19F, 6B, 14, $23 \mathrm{~F}$ (in blue and green colors); non-PCV10 serotypes: 19A, 15A, 23A (in red, orange, and yellow colors) 

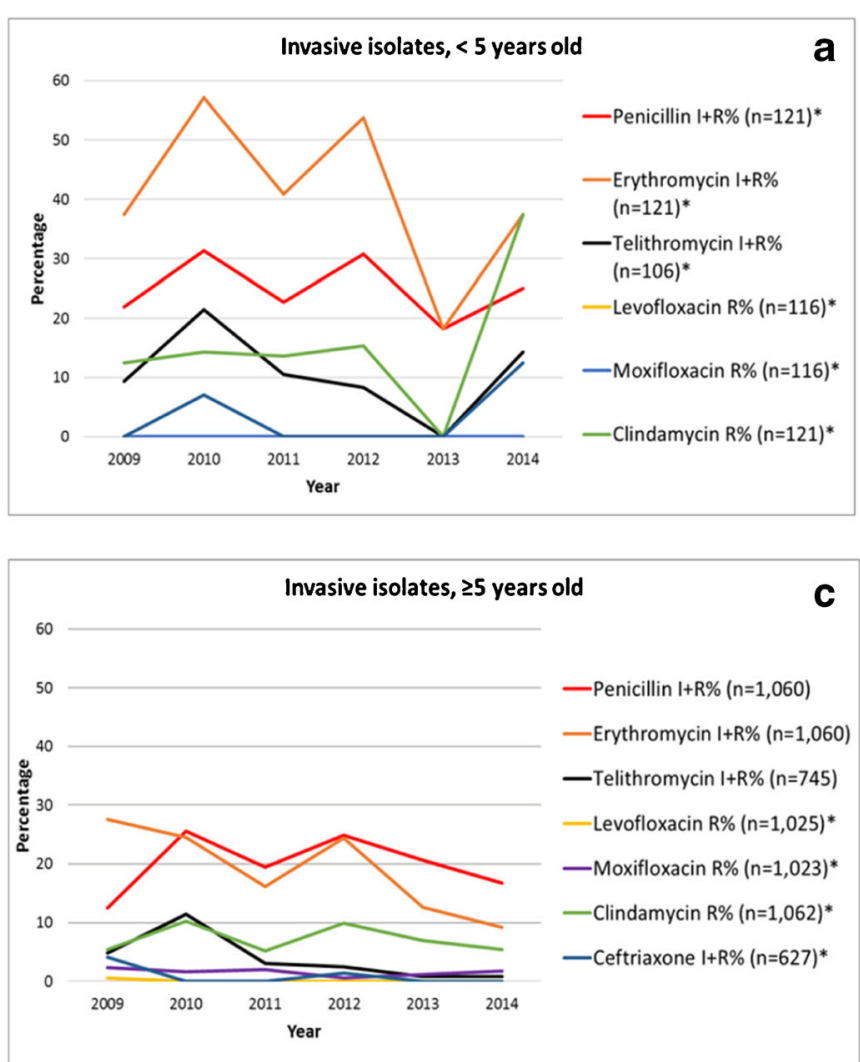

Fig. 4 a The proportion of non-susceptible invasive Streptococcus pneumoniae isolates in the $<5$ years age group by year. $\mathbf{b}$ The proportion of multidrug-resistant $(M D R)$ and non-multidrug-resistant (non-MDR) invasive $S$. pneumoniae isolates in the $<5$ years age group. c The

\section{Antimicrobial susceptibility of non-invasive isolates}

The non-susceptibility of the non-invasive isolates decreased significantly for all antimicrobial classes in the $<5$ years age group during the 6-year study period (Fig. 2a). The $p$-values were as follows: penicillin $p=0.001$, erythromycin $p<0.001$, clindamycin $p=0.002$, tetracycline $p=0.003$, and trimethoprim-sulfamethoxazole $p<0.001$. The proportion of penicillin non-susceptible isolates decreased from $26 \%(56 / 220)$ in 2009 to $13 \%(15 / 119)$ in 2014 and erythromycin nonsusceptible isolates from $37 \%(81 / 220)$ to $16 \%(19 / 119)$, respectively. The trends for non-susceptibility to all other antimicrobials followed a similar pattern. At the same time, the

Table 1 Non-invasive Streptococcus pneumoniae isolate numbers from each study year divided into the younger $(<5$ years) and older ( $\geq 5$ years) age groups

\begin{tabular}{lllllll}
\hline Number & 2009 & 2010 & 2011 & 2012 & 2013 & 2014 \\
\hline$<5$ years & 221 & 203 & 209 & 162 & 161 & 119 \\
$\geq 5$ years & 374 & 315 & 306 & 336 & 321 & 313 \\
Total & 595 & 518 & 515 & 498 & 482 & 432 \\
\hline
\end{tabular}
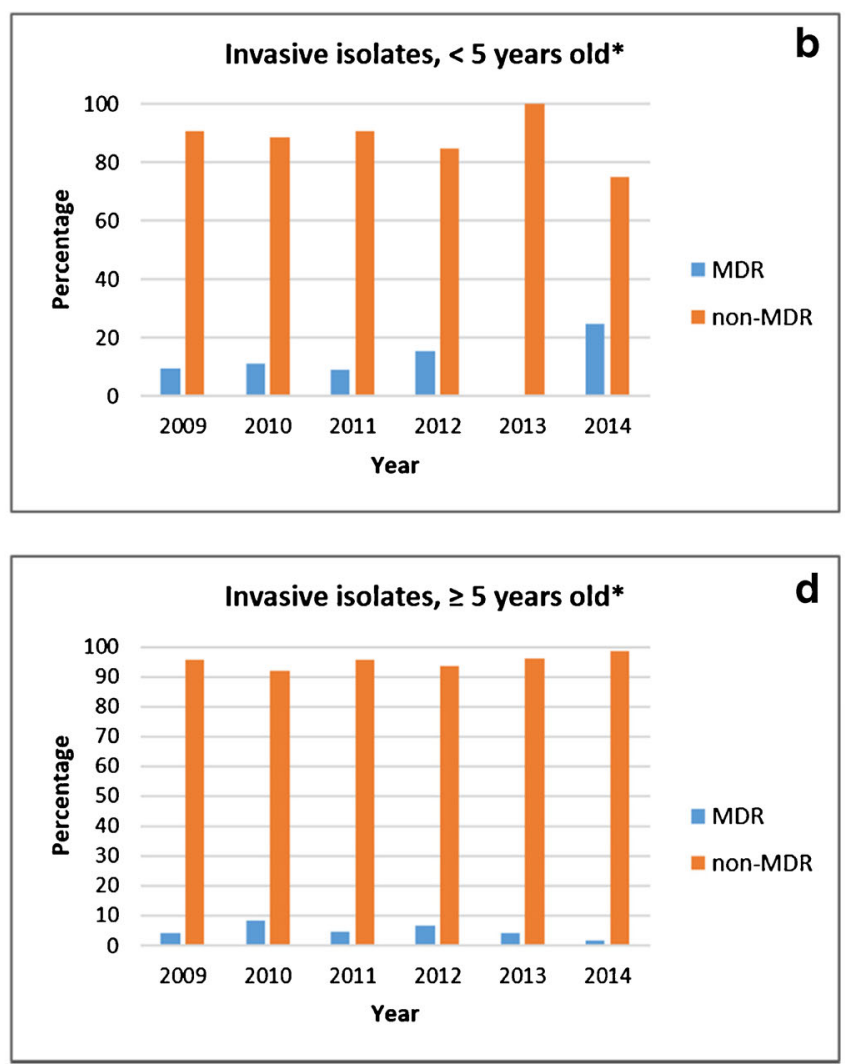

proportion of non-susceptible invasive $S$. pneumoniae isolates in the $\geq 5$ years age group by year. $\mathbf{d}$ The proportion of multidrug-resistant $(M D R)$ and non-multidrug-resistant (non-MDR) invasive $S$. pneumoniae isolates in the $\geq 5$ years age group ( $*=$ no statistical significance)

percentage of non-invasive MDR isolates in the $<5$ years age group declined significantly from $22 \%$ (49/220) in 2009 to $6 \%$ (7/119) in 2014 ( $p<0.001)$ (Fig. 2b). The percentage of penicillin-resistant $(\mathrm{MIC}>2 \mathrm{mg} / \mathrm{L})$ isolates remained low, around $0.9 \%$ annually.

Among the non-invasive isolates recovered from the $\geq 5$ years age group, the changes in antimicrobial susceptibilities are shown in Fig. 2c. The proportion of isolates nonsusceptible to erythromycin and trimethoprim-sulfamethoxazole decreased significantly when comparing years 2009 and 2014. Erythromycin non-susceptibility was $24 \%(90 / 374)$ in 2009 and $18 \%(56 / 313)$ in $2014(p=0.006)$. Trimethoprimsulfamethoxazole non-susceptibility was $23 \%(86 / 373)$ in

Table 2 Invasive Streptococcus pneumoniae isolate numbers from each study year divided into the younger $(<5$ years $)$ and older ( $\geq 5$ years) age groups

\begin{tabular}{lrrrrrr}
\hline Number & 2009 & 2010 & 2011 & 2012 & 2013 & 2014 \\
\hline$<5$ years & 33 & 37 & 27 & 13 & 13 & 8 \\
$\geq 5$ years & 195 & 200 & 162 & 182 & 184 & 200 \\
Total & 228 & 237 & 189 & 195 & 197 & 208 \\
\hline
\end{tabular}


2009 and $18 \%(55 / 311)$ in $2014(p<0.001)$. In this age group, the proportion of MDR isolates remained stable during the study period $(12 \% ; 43 / 373$ in 2009 and $11 \% ; 34 / 313$ in 2014, Fig. 2d). The proportion of penicillin-resistant isolates was approximately $0.8 \%$ annually.

\section{Serotype distribution of non-invasive MDR isolates}

We gathered all available non-invasive MDR isolates for serotyping ( $n=84,15 \%$ of all MDR isolates; yearly, 10 $20 \%$ of the isolates were stored by deep-freezing). Fortyfour $(52 \%)$ isolates were from patients $<5$ years old and 40 $(48 \%)$ isolates were from patients $\geq 5$ years old. Both PCV10 serotypes $(19 \mathrm{~F}, 6 \mathrm{~B}, 14,23 \mathrm{~F})$ and non-PCV10 serotypes (19A, 15A, 23A) were discovered among these isolates (Fig. 3). The proportion of PCV10 serotypes declined from $82 \%(18 / 22)$ in 2009 to $33 \%(2 / 6)$ in 2014 $(p<0.001)$, while the number of non-PCV10 serotypes did not change.

\section{Antimicrobial susceptibility of invasive isolates}

The number of invasive isolates in the $<5$ years age group was small $(n=131)$ and no statistically significant changes were observed between the study years when comparing antimicrobial susceptibilities or proportions of MDR isolates (Fig. 4a, b). There were no penicillin-resistant isolates in this group. The proportion of MDR isolates was 9.4\% (3/32) in 2009 and $25 \%(2 / 8)$ in 2014 , but the difference was not statistically significant due to the low isolates number (Fig. 4b).

Among invasive isolates in the $\geq 5$ years age group, nonsusceptibility decreased significantly for erythromycin $(p<0.001)$ and telithromycin $(p<0.001)$ when comparing years 2009 and 2014 (Fig. 4c). For all other antimicrobial classes, no significant decreasing trends were detected. The annual proportion of penicillin-resistant isolates was $0.2 \%$ during the study period. The proportion of MDR isolates decreased from $4.3 \%(8 / 184)$ in 2009 to $1.6 \%$ (3/184) in 2014, but the change was not statistically significant (Fig. 4d).

\section{Serotype distribution of invasive isolates}

In the $<5$ years age group, the proportion of PCV10 serotypes decreased among invasive isolates from 70\% (21/30) in 2009 to $13 \%(1 / 8)$ in $2014(p<0.001)$, while the number of nonPCV10 serotype isolates remained stable (Fig. 5a). Twelve different non-PCV10 serotypes were observed and, of these, serotype 19A was the most frequent, representing 38\% (15/39) of all non-PCV10 serotypes in the study years. After serotype $19 \mathrm{~A}$, serotypes $3(13 \% ; 5 / 39)$ and $6 \mathrm{~A}(13 \% ; 5 / 39)$ were the second most frequent. In the $\geq 5$ years age group, the proportion of PCV10 serotype isolates decreased from 63\% (112/ $177)$ in 2009 to $28 \%(52 / 183)$ in $2014(p<0.001)$. At the same time, the number of non-PCV10 serotype isolates increased from 65 (37\% of 177 isolates) in 2009 to 131 ( $72 \%$ of 183 isolates) in $2014(p<0.001)$ (Fig. 5b). In this age group, 32 different non-PCV10 serotypes were observed in the study years. The three most frequent non-PCV10 serotypes were serotypes $3(23 \% ; 115 / 501), 22 \mathrm{~F}(16 \% ; 79 / 501)$, and $19 \mathrm{~A}(12 \% ; 61 / 501)$. The trend for all three of these serotypes was increasing when comparing years 2009 and 2014.

\section{Discussion}

We studied the antimicrobial susceptibility of invasive and non-invasive pneumococcal isolates during 2009-2014 in the Helsinki Metropolitan Area, Finland, and found a significant decrease in non-susceptibility, especially in noninvasive isolates, after the introduction of PCV10 into the

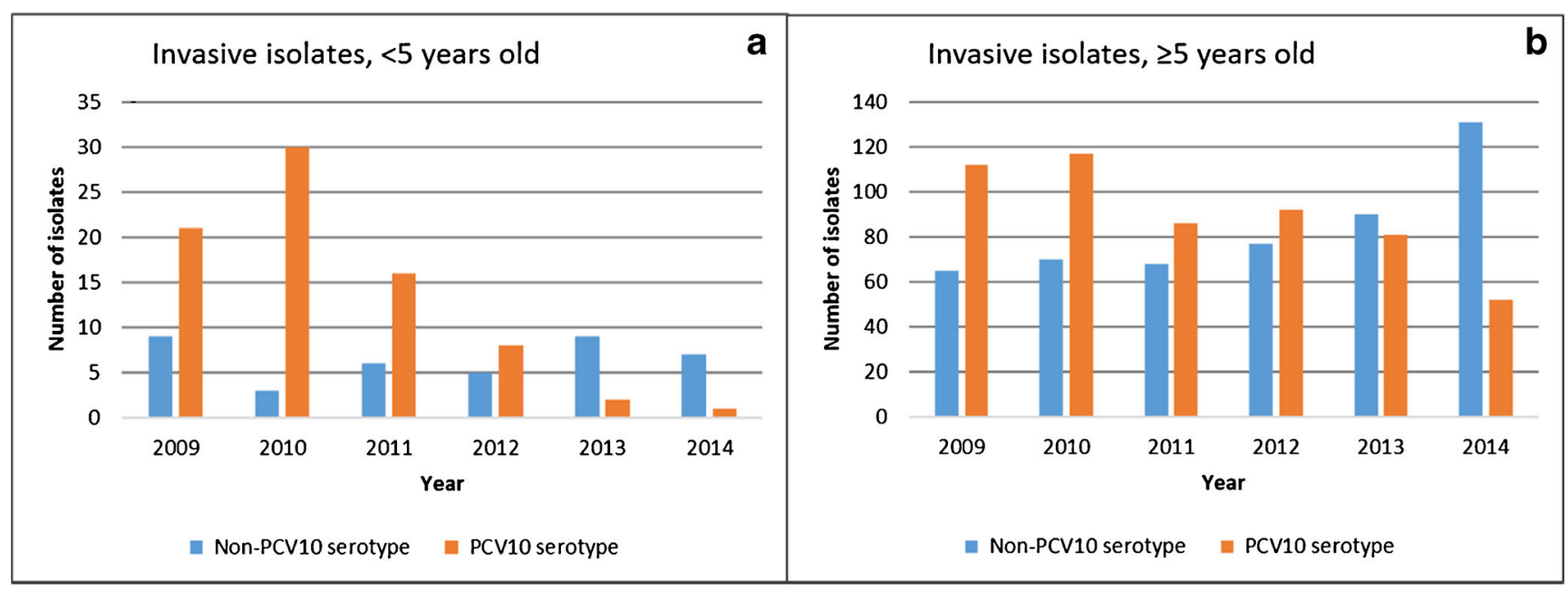

Fig. 5 a The number of invasive isolates in the $<5$ years age group by serotype group and study year. $\mathbf{b}$ The number of invasive isolates in the $\geq 5$ years age group by serotype group and study year 
national childhood vaccination program in 2010. Before PCV10 introduction, pneumococcal antimicrobial resistance was highest in non-invasive isolates of children $<5$ years old; $22 \%$ of the isolates were MDR. In 2014, four years after PCV10 introduction, non-susceptibility for all four tested antimicrobial classes (penicillin, macrolides, tetracycline, and trimethoprim-sulfamethoxazole) in these isolates had decreased and only $6 \%$ were MDR. This indicates a beneficial impact of the PCV10 vaccination. The annual percentage of penicillin resistance (MIC $>2 \mathrm{mg} / \mathrm{L}$ ) was low $(0.9 \%)$ compared to many other countries in Europe [18].

Hauser et al. [19] studied the impact of PCV7 and PCV13 vaccines on the pneumococcal antimicrobial susceptibility in Switzerland. They also found that antimicrobial nonsusceptibility was highest in children $<5$ years old and vaccine introduction caused improvement of pneumococcal penicillin, erythromycin, and trimethoprim-sulfamethoxazole susceptibility in this age group. In a Brazilian study, PCV10 vaccination improved pneumococcal penicillin susceptibility in all age groups and, additionally, susceptibility to trimethoprimsulfamethoxazole in the $<15$ years age group [20]. However, comparisons to our study are not straightforward, as the study from Brazil described the susceptibility of only 259 invasive isolates.

In this study, non-susceptibility of non-invasive isolates for erythromycin, tetracycline, and trimethoprim-sulfamethoxazole decreased also in the $\geq 5$ years age group. Although the susceptibility to penicillin did not change during the study period, these changes probably reflect the indirect effect of the PCV10 vaccine on pneumococcal antimicrobial susceptibilities among older patients.

The antimicrobial susceptibility data used in this study were comprehensive and covered both invasive and noninvasive isolates collected at the HUSLAB from the Helsinki Metropolitan Area, where almost a third of the Finnish population lives. In the Hospital District of Helsinki and Uusimaa, the methods and guidelines to diagnose and treat pneumococcal diseases have remained the same during the studied years and, thus, we conclude that our results likely reflect true changes caused by the vaccination, rather than changes in diagnostic or clinical practices. After the start of PCV10 vaccination, the outpatient antimicrobial purchase numbers have also decreased in the vaccinated child population in Finland [21]. In addition to directly reducing or eliminating pneumococcal disease caused by antimicrobial nonsusceptible PCV10 serotypes, the subsequent reduction in antimicrobial consumption and, hence, reduced antimicrobial selection pressure may also discourage the development of resistance in pneumococci in the human respiratory tract. Both invasive and non-invasive pneumococcal isolate numbers declined in the $<5$ years age group, suggesting reduced disease burden.
Our serotype data of invasive isolates were comprehensive and collected continuously by the National Institute for Health and Welfare for surveillance. In the younger age group ( $<5$ years), the PCV10 serotypes almost disappeared, but the number of non-PCV10 serotype isolates remained stable throughout the study period. dos Santos et al. have described similar results in patients $<2$ years old in Brazil after PCV10 introduction, but the follow-up time in that study was only 2 years [20]. In our study, the PCV10 serotypes decreased also among invasive isolates from older patients ( $\geq 5$ years). Since PCV vaccination has not been routinely used in Finland for the elderly population, these patients may represent mostly the unvaccinated population and, thus, the reduction in IPD was most likely due to herd protection [22]. At the same time, the number of non-PCV10 serotype isolates increased in this age group, resulting in a stable total number of invasive isolates during the whole study period. This rise was most pronounced in 2014. A longer surveillance will show if the change continues.

To estimate reliably changes in the serotype distribution of non-invasive MDR isolates, more data would be needed, since our subset represented only $10-20 \%$ of the MDR isolates. However, it offers a view of possible changes that may have taken place in the serotype distribution of non-invasive isolates since the large-scale use of PCV10. Our findings suggest that PCV10 serotypes have decreased also among these isolates. It will be interesting to follow up how long this may last and if the proportion of resistant non-PCV10 serotype isolates, such as 19A, will increase in the future. Increased incidence of serotype 19A and other non-PCV7 serotype isolates has been shown after PCV7 introduction in the USA, the Netherlands, and many other countries [23, 24]. In France, the prevalence of antimicrobial-resistant pneumococci decreased after PCV13 introduction and was mainly driven by the decline of the serotype 19A [25].

Finally, we conclude that, due to PCV10 vaccination, invasive pneumococcal infections have almost disappeared among patients $<5$ years old and the non-susceptibility of non-invasive pneumococcal isolates in this age group has decreased for all antimicrobial classes. Serotype replacement phenomenon has presumably begun in invasive isolates among patients $\geq 5$ years old, but further surveillance is needed.

Acknowledgments Jukka Ollgren: support in statistics.

\section{Compliance with ethical standards}

Funding No funding was received from any source with an interest in our findings.

Conflict of interest The authors declare that they have no conflict of interest. 
Ethical approval This article does not contain any studies with human participants or animals performed by any of the authors.

Informed consent Informed consent was not needed since this was an epidemiological register study approved by the Helsinki University Hospital.

\section{References}

1. Mitchell TJ (2003) The pathogenesis of streptococcal infections: from tooth decay to meningitis. Nat Rev Microbiol 1:219-230

2. Austrian R, Gold J (1964) Pneumococcal bacteremia with especial reference to bacteremic pneumococcal pneumonia. Ann Intern Med 60(5):759-776

3. World Health Organization (WHO) (2014) Antimicrobial resistance: global report on surveillance 2014. WHO, Geneva, Switzerland. Available online at: http://www.who.int/ drugresistance/documents/surveillancereport/en/

4. Siira L, Jalava J, Kaijalainen T, Ollgren J, Lyytikäinen O, Virolainen A (2014) Antimicrobial resistance in relation to seroand genotypes among invasive Streptococcus pneumoniae in Finland, 2007-2011. Microb Drug Resist 20(2):124-130

5. Siira L, Rantala M, Jalava J, Hakanen AJ, Huovinen P, Kaijalainen T, Lyytikäinen O, Virolainen A (2009) Temporal trends of antimicrobial resistance and clonality of invasive Streptococcus pneumoniae isolates in Finland, 2002 to 2006. Antimicrob Agents Chemother 53(5):2066-2073

6. Bentley SD, Aanensen DM, Mavroidi A, Saunders D, Rabbinowitsch E, Collins M, Donohoe K, Harris D, Murphy L, Quail MA, Samuel G, Skovsted IC, Kaltoft MS, Barrell B, Reeves PR, Parkhill J, Spratt BG (2006) Genetic analysis of the capsular biosynthetic locus from all 90 pneumococcal serotypes. PLoS Genet 2(3):e31

7. Black S, Shinefield H, Fireman B, Lewis E, Ray P, Hansen JR, Elvin L, Ensor KM, Hackell J, Siber G, Malinoski F, Madore D, Chang I, Kohberger R, Watson W, Austrian R, Edwards K (2000) Efficacy, safety and immunogenicity of heptavalent pneumococcal conjugate vaccine in children. Northern California Kaiser Permanente Vaccine Study Center Group. Pediatr Infect Dis J 19(3):187-195

8. Whitney CG, Farley MM, Hadler J, Harrison LH, Bennett NM, Lynfield R, Reingold A, Cieslak PR, Pilishvili T, Jackson D, Facklam RR, Jorgensen JH, Schuchat A; Active Bacterial Core Surveillance of the Emerging Infections Program Network (2003) Decline in invasive pneumococcal disease after the introduction of protein-polysaccharide conjugate vaccine. N Engl J Med 348(18): 1737-1746

9. Eskola J, Kilpi T, Palmu A, Jokinen J, Haapakoski J, Herva E, Takala A, Käyhty H, Karma P, Kohberger R, Siber G, Mäkelä PH; Finnish Otitis Media Study Group (2001) Efficacy of a pneumococcal conjugate vaccine against acute otitis media. $\mathrm{N}$ Engl $\mathrm{J}$ Med 344(6):403-409

10. Black SB, Shinefield HR, Ling S, Hansen J, Fireman B, Spring D, Noyes J, Lewis E, Ray P, Lee J, Hackell J (2002) Effectiveness of heptavalent pneumococcal conjugate vaccine in children younger than five years of age for prevention of pneumonia. Pediatr Infect Dis J 21(9):810-815

11. Moore MR, Link-Gelles R, Schaffner W, Lynfield R, Lexau C, Bennett NM, Petit S, Zansky SM, Harrison LH, Reingold A, Miller L, Scherzinger K, Thomas A, Farley MM, Zell ER, Taylor TH Jr, Pondo T, Rodgers L, McGee L, Beall B, Jorgensen JH, Whitney CG (2015) Effect of use of 13-valent pneumococcal conjugate vaccine in children on invasive pneumococcal disease in children and adults in the USA: analysis of multisite, populationbased surveillance. Lancet Infect Dis 15(3):301-309

12. Palmu AA, Jokinen J, Borys D, Nieminen H, Ruokokoski E, Siira L, Puumalainen T, Lommel P, Hezareh M, Moreira M, Schuerman L, Kilpi TM (2013) Effectiveness of the ten-valent pneumococcal Haemophilus influenzae protein D conjugate vaccine (PHiDCV10) against invasive pneumococcal disease: a cluster randomised trial. Lancet 381(9862):214-222

13. Palmu AA, Jokinen J, Nieminen H, Syrjänen R, Ruokokoski E, Puumalainen T, Moreira M, Schuerman L, Borys D, Kilpi TM (2014) Vaccine effectiveness of the pneumococcal Haemophilus influenzae protein D conjugate vaccine (PHiD-CV10) against clinically suspected invasive pneumococcal disease: a clusterrandomised trial. Lancet Respir Med 2(9):717-727

14. Jokinen J, Rinta-Kokko H, Siira L, Palmu AA, Virtanen MJ, Nohynek H, Virolainen-Julkunen A, Toropainen M, Nuorti JP (2015) Impact of ten-valent pneumococcal conjugate vaccination on invasive pneumococcal disease in Finnish children-a population-based study. PLoS One 10(3):e0120290

15. Jokinen J, Nuorti P, Palmu A, Virolainen-Julkunen A, Nohynek H, Virtanen M, Siira L, Lahdenkari M, Ruutu P, Kilpi T (2012) Monitoring the effectiveness of Finnish national vaccination programme (NVP) of the 10-valent pneumococcal conjugate vaccine (PCV10). In: Proceedings of the 8th International Symposium on Pneumococci and Pneumococcal Diseases (ISPPD-8), Iguaçu Falls, Brazil, 11-15 March 2012. Poster A-428-0023-00373

16. McAvin JC, Reilly PA, Roudabush RM, Barnes WJ, Salmen A, Jackson GW, Beninga KK, Astorga A, McCleskey FK, Huff WB, Niemeyer D, Lohman KL (2001) Sensitive and specific method for rapid identification of Streptococcus pneumoniae using real-time fluorescence PCR. J Clin Microbiol 39(10):3446-3451

17. Siira L, Kaijalainen T, Lambertsen L, Nahm MH, Toropainen M, Virolainen A (2012) From Quellung to multiplex PCR, and back when needed, in pneumococcal serotyping. J Clin Microbiol 50(8): 2727-2731

18. European Centre for Disease Prevention and Control (ECDC). Antimicrobial resistance. Available online at: http://ecdc.europa. $\mathrm{eu} / \mathrm{en} / \mathrm{health}$ topics/antimicrobial-resistance-and-consumption/ antimicrobial resistance/database/Pages/map reports.aspx

19. Hauser C, Kronenberg A, Allemann A, Mühlemann K, Hilty M (2016) Serotype/serogroup-specific antibiotic non-susceptibility of invasive and non-invasive Streptococcus pneumoniae, Switzerland, 2004 to 2014. Euro Surveill 21(21)

20. dos Santos SR, Passadore LF, Takagi EH, Fujii CM, Yoshioka CR, Gilio AE, Martinez MB (2013) Serotype distribution of Streptococcus pneumoniae isolated from patients with invasive pneumococcal disease in Brazil before and after tenpneumococcal conjugate vaccine implementation. Vaccine 31(51): 6150-6154

21. Palmu AA, Jokinen J, Nieminen H, Rinta-Kokko H, Ruokokoski E, Puumalainen T, Borys D, Lommel P, Traskine M, Moreira M, Schuerman L, Kilpi TM (2014) Effect of pneumococcal Haemophilus influenzae protein D conjugate vaccine (PHiDCV10) on outpatient antimicrobial purchases: a double-blind, cluster randomised phase 3-4 trial. Lancet Infect Dis 14(3):205-212

22. Bonten MJ, Huijts SM, Bolkenbaas M, Webber C, Patterson S, Gault S, van Werkhoven CH, van Deursen AM, Sanders EA, Verheij TJ, Patton M, McDonough A, Moradoghli-Haftvani A, Smith H, Mellelieu T, Pride MW, Crowther G, Schmoele-Thoma B, Scott DA, Jansen KU, Lobatto R, Oosterman B, Visser N, Caspers E, Smorenburg A, Emini EA, Gruber WC, Grobbee DE (2015) Polysaccharide conjugate vaccine against pneumococcal pneumonia in adults. N Engl J Med 372(12):1114-1125

23. van Deursen AMM, van Mens SP, Sanders EAM, Vlaminckx BJM, de Melker HE, Schouls LM, de Greeff SC, van der Ende A; Invasive Pneumococcal Disease Sentinel Surveillance Laboratory 
Group (2012) Invasive pneumococcal disease and 7-valent pneumococcal conjugate vaccine, the Netherlands. Emerg Infect Dis 18(11):1729-1737

24. Kim L, McGee L, Tomczyk S, Beall B (2016) Biological and epidemiological features of antibiotic-resistant Streptococcus pneumoniae in pre- and post-conjugate vaccine eras: a United States perspective. Clin Microbiol Rev 29(3):525-552
25. Janoir C, Lepoutre A, Gutmann L, Varon E (2016) Insight into resistance phenotypes of emergent non 13-valent pneumococcal conjugate vaccine type pneumococci isolated from invasive disease after 13-valent pneumococcal conjugate vaccine implementation in France. Open Forum Infect Dis 3(1):ofw020 